\title{
Identifying and analyzing the construction and effectiveness of offensive plays in basketball by using systematic observation
}

\author{
Jordi Fernandez and Oleguer Camerino \\ INEFC-University of Lleida, Lleida, Spain \\ M. Teresa Anguera \\ University of Barcelona, Barcelona, Spain \\ AND \\ GUDBERG K. JONSSON \\ University of Iceland, Reykjavik, Iceland \\ and University of Aberdeen, Aberdeen, Scotland
}

\begin{abstract}
In the field of sports research, there is a growing need for the rigorous collection of data that provide empirical evidence about the complex reality they refer to. Although sports psychology research has advanced considerably in recent years, in both extent and quality, one area of research that remains relatively unexplored is the dynamics of the sports group and the influence of the group on its members (George \& Feltz, 1995; Widmeyer, Brawley, \& Carron, 1992). Key aspects in this regard include the presence of regularities that are not detectable through visual inference or traditional methods of data analysis, the lack of standard observation instruments, and, assuming priority, the need to develop powerful, computerized coding systems, all of which must form part of an approach that is suitable for natural and habitual contexts. The present study is part of a broader research project concerning ACB teams (first Spanish basketball division) and considers the interaction context before teams try to score (where this is understood as how teams create scoring opportunities) as the core aspect that links team play. This investigation proposes a new model of analysis for studying the effectiveness and construction of offensive basketball plays in order to identify their outcomes, thus providing coaches with an important device for improving or consolidating them.
\end{abstract}

Team sports are now the focus of many studies in different fields of science. As a whole, these sports are watched and practiced by millions of people and are also observed by companies as a model with which to improve teamwork. A common feature is that each of them comprises a complex network of strategic possibilities, where the most noticeable part is the result. Although this is usually measured in terms of victory or defeat, this success or failure depends partly on the combination of all the work done by the different branches of the team structure and the requirements of each competitive situation that take priority.

Each team or club has its own hierarchy, and one of the fundamental aspects of this is that coaches are an active part of the process; this means that all the responsibility for what takes place within this process is placed on them. Thus, they have to be trained to store enough knowledge to take part during the dynamic process, which is always changing, and this is where their day-to-day planning of the work comes into play.
Both researchers and coaches have shown particular interest in analyzing all the actions involved in making team sports efficient. This may be done with the aim of developing a deeper understanding of the game's content and its logic or of creating practical training situations that favor competitive efficiency (Garganta, 2001).

Teams are the result of a large structure, the success of which depends on the work of each of its constituent parts. As has already been pointed out, it is coaches who are responsible for ensuring that the system works properly on all levels. Their main job is to delegate all the different tasks within the group, and from these tasks they will take charge of the most important details so as to make the decisions needed for the team to succeed. This is why coaches have to receive all the necessary information and why this information needs to be of high quality. All this will help them to develop as team leaders.

Decision strategies differ with regard to the degree of quantitative and qualitative reasoning used. In general, 
strategies that involve summing, subtracting, and/or multiplying values, as well as counting, are considered to be quantitative. In contrast, strategies that simply compare values are regarded as qualitative (Riedl, Brandstätter, \& Roithmayr, 2008). Traditional analytic methods have used frequency of event occurrence as their index of performance - for example, recording the number of passes made from particular zones or how many times a team makes a mistake. However, if one accepts that sports performance consists of a complex series of interrelationships between wide arrays of performance variables, simple frequency data can provide only a relatively superficial view of performance. There is thus a growing demand for data analysis methods or techniques that can generate more complete and, therefore, more complex quantitative representations of performance (Anguera \& Jonsson, 2003).

This lack of research is surprising given the predominance of the group in sports and the importance that is attached to participation in, and performance of, sports teams cross-culturally. That sports psychology should focus upon the individual cannot be disputed, but ignoring the potential influence of the group on such aspects as satisfaction, motivation, psychological well-being, and, ultimately, both individual and group performance may be to overlook an important component of the psychology of sports (Prapavessis \& Carron, 1996).

One group factor that has been suggested to be important in sports teams is Bandura's $(1982,1986)$ concept of collective efficacy, which refers to the application of efficacy at a group level. Bandura's (1977) original theorizing on the concept of efficacy focused upon selfefficacy - that is, the belief in one's ability to achieve a desired outcome - and predicted that, given adequate incentive and the requisite skill level, self-efficacy will determine the challenges that individuals select, the effort they exert on tasks, and their persistence when faced with failure. Indeed, such predictions have consistently been supported in both the general psychology and sports-specific literature (Feltz, 1992). However, stemming from the realization that much of human endeavor is group based, in which the attainment of desired outcomes is determined by the performance of the group as a whole, Bandura $(1982,1986,1997)$ extended the influence of efficacy to the group.

\section{METHOD}

The observational methodology used had the rigor and flexibility needed to study the episodes and different configurations of play just as they occurred.

One of the concerns of this project is to study the hidden structures underlying an interactive situation such as a game of basketball. In the approach taken here, we are concerned with the way in which temporal patterns are able to reveal those aspects of social interaction that are not immediately observable, and we consider every interactive flow to be governed by behavioral structures of varying stability that can be visualized by detecting hidden temporal patterns such as T-patterns. These temporal patterns can be detected by means of the powerful algorithm of the THEME soft- ware, developed by Magnusson (1996, 2000), which imports data obtained by SOF-CODER. This constitutes the second aim of the present study.

The use of T-patterns and THEME has proven to be extraordinarily productive for the study of the many different aspects or fields of social interaction, as has been illustrated by research into team sports (Anguera \& Jonsson, 2003; Bloomfield, Jonsson, Polman, Houlahan, \& O'Donoghue, 2005; Borrie, Jonsson, \& Magnusson, 2001, 2002; Jonsson, Bjarkadottir, Gislason, Borrie, \& Magnusson, 2003).

\section{Design}

The observational design (Anguera, Blanco-Villaseñor, \& Losada, 2001) was nomothetic (several games), point (one game for each pair of teams, and within-session recording throughout the game), and multidimensional (the dimensions correspond with the criteria of the observation instrument).

The use of this nomothetic/point/multidimensional (N/P/M) design determines a series of decisions regarding participants, instruments (for observation-i.e., the SOBL-1 - and recording) and procedure.

\section{Participants}

This study is part of a broader research project concerning games of one selected team playing against different teams at the same level of competition - in this case, from Spain's first basketball division (ACB). Here, we selected five games played by the Ricoh Manresa team in the Spanish ACB League during the 2007-2008 season. A common feature of these games is that they were all victories for the selected team during the first round of the league. Table 1 shows in italics the team observed in each game.

\section{Instruments}

Observation instrument. The ongoing development of observation instruments has enabled us to conduct detailed studies of the dynamics of play in different team sports (soccer, basketball, handball, and volleyball). The observation instrument chosen for the present study was the SOBL-2, which combines field formats with category systems (Jonsson et al., 2006). The SOBL-2 was chosen because its criteria or dimensions - the result or score, the actions of the team, the zones on the court, and laterality — are in line with the objectives of this study.

This new instrument (SOBL-2) detects 26 zones on the basketball court in order to analyze the actions that provide scoring opportunities. By analyzing the zones of the last two passes and the last two receptions before a team tries to score, we can learn about the effectiveness of basketball plays. This additional information can also enhance performance through improved quality of performer feedback, provided that the feedback is given in appropriate forms.

The SOBL-2 (see Table 2) is consistent with the proposed observational design, which is multidimensional in nature and has the following structure of criteria and categories. The changing criteria (laterality, zone, action, court, and game) are applied in a continuous recording throughout the observation of the whole game. Each of them gives rise to respective category systems that fulfill the conditions of exhaustiveness and mutual exclusivity (E/ME).

Table 1

List of Basketball Games Observed

\begin{tabular}{rrll}
\hline \multicolumn{2}{c}{ Result } & Venue & \multicolumn{1}{c}{ Teams } \\
\hline Won & $87-79$ & home & Manresa-Menorca \\
& $73-90$ & away & Fuenlabrada-Manresa \\
& $86-79$ & home & Manresa -Estudiantes \\
& $89-81$ & home & Manres $a-$ Unicaja \\
& $78-81$ & away & Leon-Manresa \\
\hline
\end{tabular}

Note-The team observed in each game is shown in italics. 
Table 2

Codes Corresponding to the Observation Instrument SOBL-2

\begin{tabular}{|c|c|c|}
\hline Laterality & $\begin{array}{l}\text { Right side } \\
\text { Right baseline } \\
\text { Center } \\
\text { Left side } \\
\text { Left baseline }\end{array}$ & $\begin{array}{l}\text { Dl } \\
\text { Df } \\
\text { C } \\
\text { Izl } \\
\text { Izf }\end{array}$ \\
\hline Zone & $\begin{array}{l}\text { Outside } \\
\text { Zone } \\
\text { Paint } \\
\text { Outside of the court }\end{array}$ & $\begin{array}{l}\mathrm{E} \\
\mathrm{Z} \\
\mathrm{P} \\
\mathrm{Fu}\end{array}$ \\
\hline Action & $\begin{array}{l}\text { Steal } \\
\text { Offensive rebound } \\
\text { Defensive rebound } \\
\text { Pass before the last one } \\
\text { Reception after P1 } \\
\text { Last pass } \\
\text { Last reception } \\
\text { Ending }\end{array}$ & $\begin{array}{l}\text { Rec } \\
\text { Rd } \\
\text { R } \\
\text { P1 } \\
\text { R1 } \\
\text { P2 } \\
\text { R2 } \\
\text { Fn }\end{array}$ \\
\hline Ending & $\begin{array}{l}\text { Make } \\
\text { Miss } \\
\text { Foul } \\
\text { And one } \\
\text { Block }\end{array}$ & $\begin{array}{l}\text { En } \\
\text { Er } \\
\mathrm{Fr} \\
\mathrm{A} 1 \\
\mathrm{Tp}\end{array}$ \\
\hline Court & $\begin{array}{l}\text { Offensive court } \\
\text { Defensive court }\end{array}$ & $\begin{array}{l}\text { Qf } \\
\text { Def }\end{array}$ \\
\hline Beginning of the play & $\begin{array}{l}\text { Side take out } \\
\text { Baseline take out } \\
\text { Initial jump } \\
\text { Free throw } \\
\text { Ball in life }\end{array}$ & $\begin{array}{l}\text { Sbo } \\
\text { Sfo } \\
\text { Si } \\
\text { Tl } \\
\text { Bj }\end{array}$ \\
\hline Game & $\begin{array}{l}\text { Static (five-on-five) } \\
\text { Fast break }\end{array}$ & $\begin{array}{l}\text { Est } \\
\text { FastB }\end{array}$ \\
\hline
\end{tabular}

The dimensions considered in this study correspond to the following criteria.

1. Action of the team observed: Here, we describe the different actions that are registered during the creation of a scoring opportunity:

P1: The pass before the last one when the observed team tries to score.

$\mathrm{R} 1$ : The reception after this $\mathrm{P} 1$.

P2: The last pass before the observed team tries to score

R2: The last reception before the observed team tries to score.

Fn: The action that means the observed team takes a shot.

Rec: The observed team steals the ball.

$\mathrm{R}$ : The observed team gets an offensive rebound.

$\mathrm{Rd}$ : The observed team gets a defensive rebound.

2. Zone (see Figure 1), determined by the court zones. These zones are limited by the basketball rules: paint (3-sec rule and a score of two points), zone (no $3-\mathrm{sec}$ rule and a score of two points), and outside (behind the three-point line and a score of three points).

3. Laterality (see Figure 2) of play, defined according to five court areas or spatial strips: right side, right baseline, center, left side, and left baseline.

4. Game. We separate the normal actions when the observed team plays five-on-five from actions when the same team plays fastbreak.

5. Court. To distinguish between the offensive court and the defensive court.

6. Endings. The different ways in which a player can obtain a result after taking a shot or lay-up: makes, mistakes, blocks, received fouls one-and-ones.

7. Beginning of plays. This is the point where we start to register each advantageous play. It can be from: the initial jump, a side or baseline take out, or a live ball.
Recording instrument. The recording instrument used was Match Vision Studio 3.0 software (Castellano, Perea, Alday, \& Hernández Mendo, 2008). This is a user-friendly tool developed to help researchers observe, codify, register, and analyze any situation that occurs in a natural or habitual context in which behaviors are spontaneous. Match Vision Studio 3.0 (Figure 3) is an interactive multimedia package that enables the digitalized recording of games to be viewed and recorded, in an avi or .mpg file, on the computer screen itself.

This is a highly flexible software package in that it enabled us, first, to introduce all the codes corresponding to each one of the dimensions of the changing criteria of the observation instrument (SOBL-2) so as to register their co-occurrence. If there was a change in any of these criteria, we froze the image and recorded this event on the screen itself.

After recording each quarter or the end of a game we obtained an Excel file (Table 3) comprising the successive configurations formed by the lines of codes that have changed, along with their temporality and duration expressed in frames ( 25 frames is equivalent to $1 \mathrm{sec}$ )

Data quality (Blanco-Villaseñor \& Anguera, 2000) was assessed by calculating the kappa coefficient using SDIS-GSEQ, Version 4.1.5, software (Bakeman \& Quera, 1992, 2001); its values were between 0.75 and 0.85 .

\section{Procedure}

The .xls files obtained from the Excel program, which provided us with the frequencies for all the events of the registered codes, were successively transformed in order to enable different treatments: first, a sequential treatment with SDIS-GSEQ, and second, with the THEME software (Table 3 ).

In order to conduct a more detailed analysis of the sequential relationship between the actions, zone, laterality, and endings, the .xls files were transformed into one of the formats required by the SDIS-GSEQ software (Bakeman \& Quera, 1992, 2001; see sample in Table 4), that of Multievent.

In order to obtain T-Patterns from .xls files of games won, these were transformed into a CSV file recognized by the THEME v.5 software (Magnusson, 1996, 2000; see sample in Table 5).

\section{RESULTS}

Given that our interest here was to study the influence of the offensive areas where the observed team creates a scoring opportunity in professional basketball, the data analysis was conducted in successive stages.

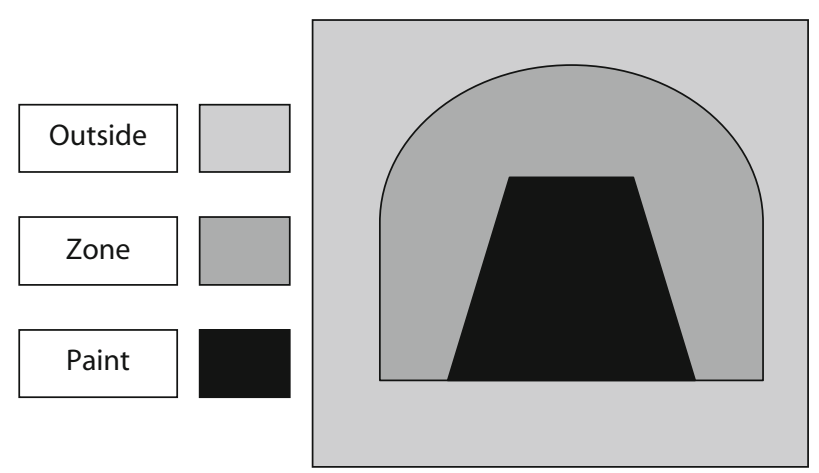

Figure 1. Example of zone criteria according to the rules of basketball. 


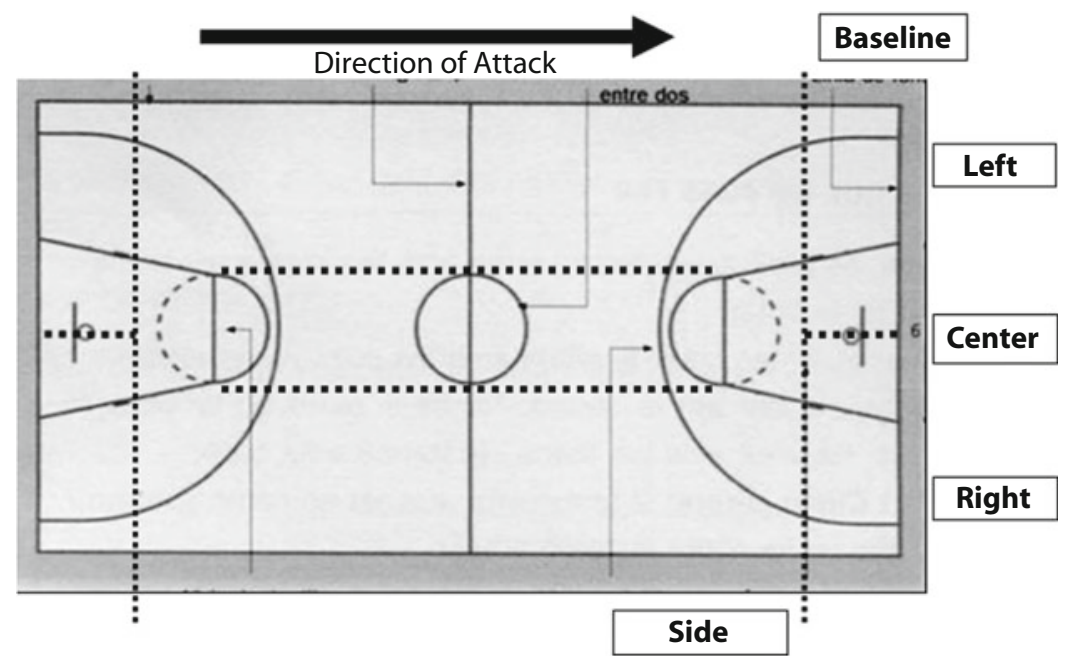

Figure 2. Laterality of the court as regards the observed team.

\section{Descriptive Analysis}

By observing the frequency of the data selected in the THEME software, which was obtained by mixing the different criteria, we can state that our observed team ends by creating shots from the outside zones. It then finishes from the paint and outside the three-point line and obtains an appropriate equilibrium enabling it to succeed in all of the five games recorded.
Four criteria were selected to analyze the quality of the team's actions, and this is why we mixed laterality, zone, action, and ending. Subsequently, we also introduced the game and court criteria. The mixing of all these criteria enables us to describe where all the actions took place and thus provide an evaluation of the observed game.

The frequency of the four mixed criteria describes the following game style (see Figure 4).

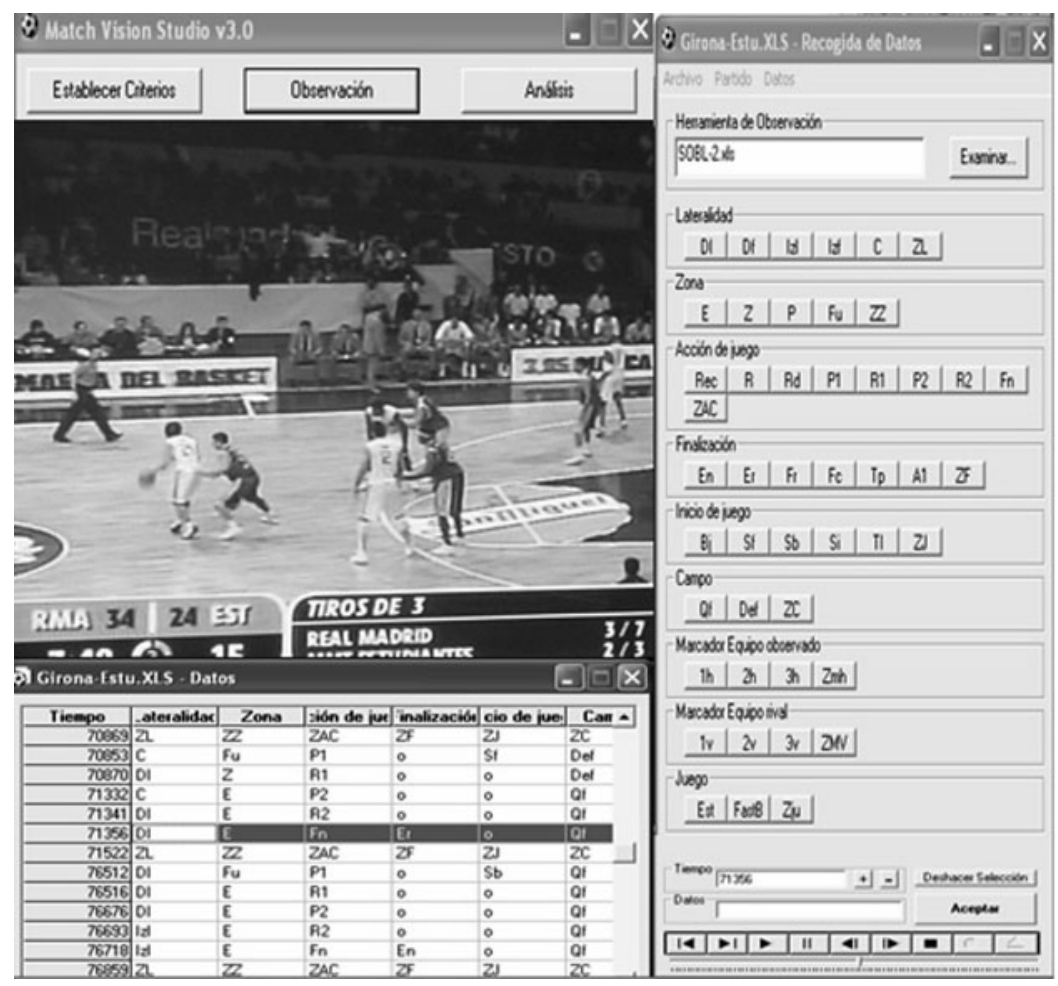

Figure 3. Recording instrument: Match Vision Studio 3.0 (Castellano, Perea, Alday, \& Hernández-Mendo, 2008). 
Table 3

Recording Obtained by Means of Match Vision Studio 3.0 (Game Between Manresa and Estudiantes)

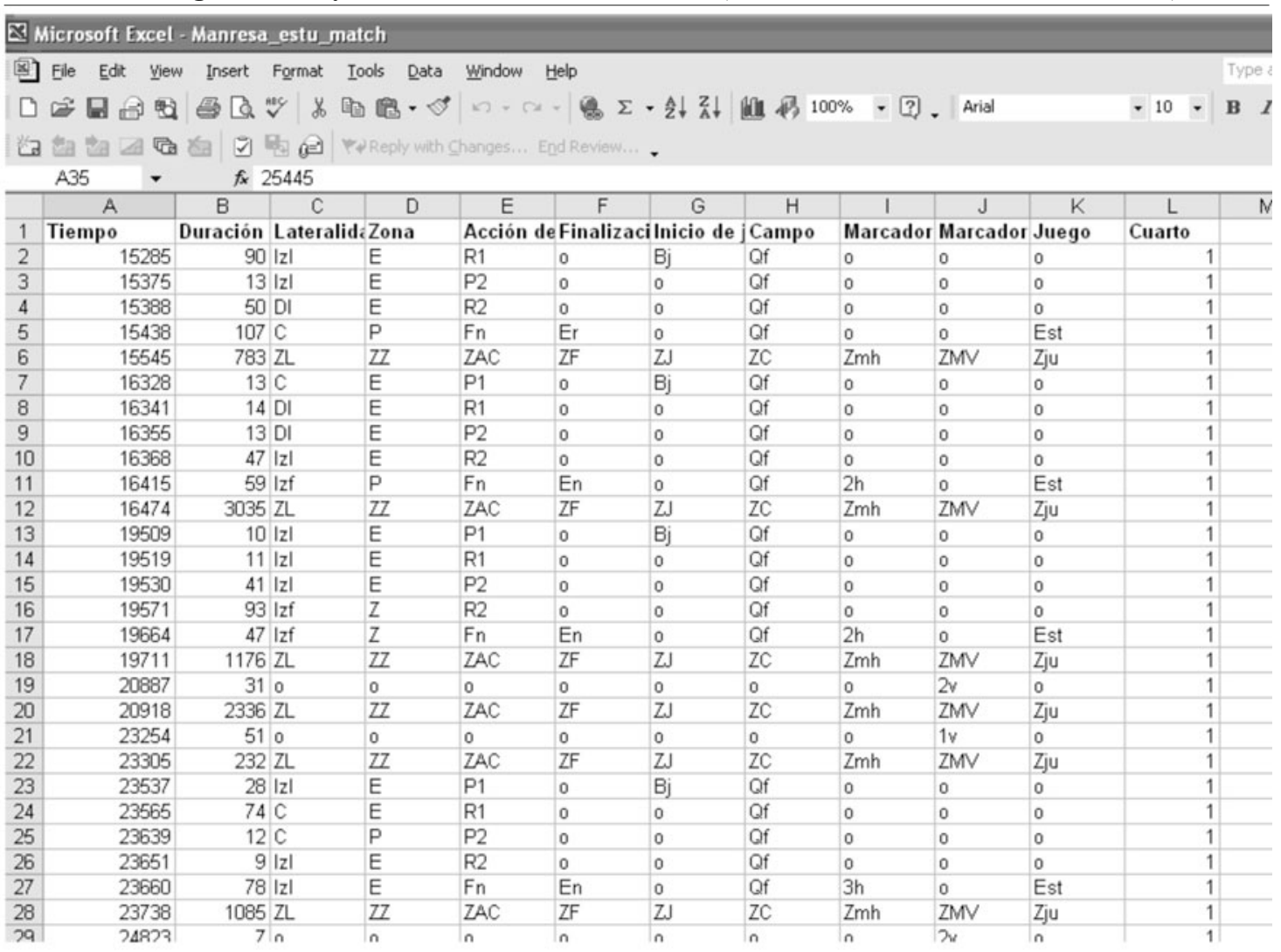

P1. Among all the registered frequencies, $\mathrm{P} 1$ is the one that most often occurs when the observed team starts each play. Most of these P1 actions take place in the outside zone with central (C) (23), right-side (Dl) (42), and leftside (Izl) laterality (35). There are some other P1s that take place in the outside zone (E) (12) or inside the paint (P) (9), but with less frequency. As regards the frequencies in the outside zone, these make sense during special plays, such as those from the baseline or sideline. The other P1, inside the paint, refers to extra pass plays, which means that the ball goes inside, closing off all assistance, and giving an extra pass that creates space in other positions.

$R 1$. All the R1 actions that occur before the ending take place mostly in the outside zone (E), and always in the right (Dl) (45) and left (Izl) side zones (56). Thus, all these actions serve to swing the ball from the middle or from side to side, as well as for hand-offs.

$P 2$. The last pass before the shot or lay-up is taken by the observed team occurs mostly in the outside zones (E): central (C) (28), right side (Dl) (46), and left side (Izl) (43). P2 also occurs a little less often in the central paint area (CP) (21). All these frequencies show that the plays created by the observed team come mostly from a swing of the ball. The ball is moved effectively in the center (C), right-side (Dl), and left-side (Izl) outside zones in order to pass the ball inside or to keep passing outside to shoot for three points. In contrast, all the P2s that occurred in the paint $(\mathrm{P})$ come from one-on-ones from the outside zones (E) (guards and forwards), closing the defense to give an open shot or a easy pass close to the rim.

$R 2$. As regards the actions that refer to last reception, the highest frequencies correspond to the outside zones (E), once again center (C) (23), right side (Dl) (33), and left side (Izl) (41). However, these are followed by plays in the inside areas, all three paint spots $(\mathrm{P})$, center paint (CP) (22), left baseline (Izf) (13), and right baseline (Df) (22). This information shows that there is a proper balance to the observed team's game.

$\mathrm{Fn}$. The last actions registered in the play as a whole show where each play culminates and the percentages (makes/misses) on each spot. Note the high percentages for shots taken on the spots close to the rim (P).

IzfP (left-baseline paint): $22 / 32=68.7 \%$

CP (center paint): $20 / 42=47.6 \%$

DfP (right-baseline paint): $13 / 22=59 \%$

IzLE (left-side outside): $6 / 18=33 \%$

CE (center outside): $12 / 22=54.5 \%$

DLE (right-side outside): $6 / 18=33 \%$

\section{Detecting Temporal Patterns}

In order to explore in greater depth the differences between the last five actions preceding an ending and the exact location where they each occur, we next conducted 
Table 4

Example of a Transformed Recording That Has Been Exported to the SDIS-GSEQ Software (Game Between Manresa and Estudiantes), With Data in Multievent Form

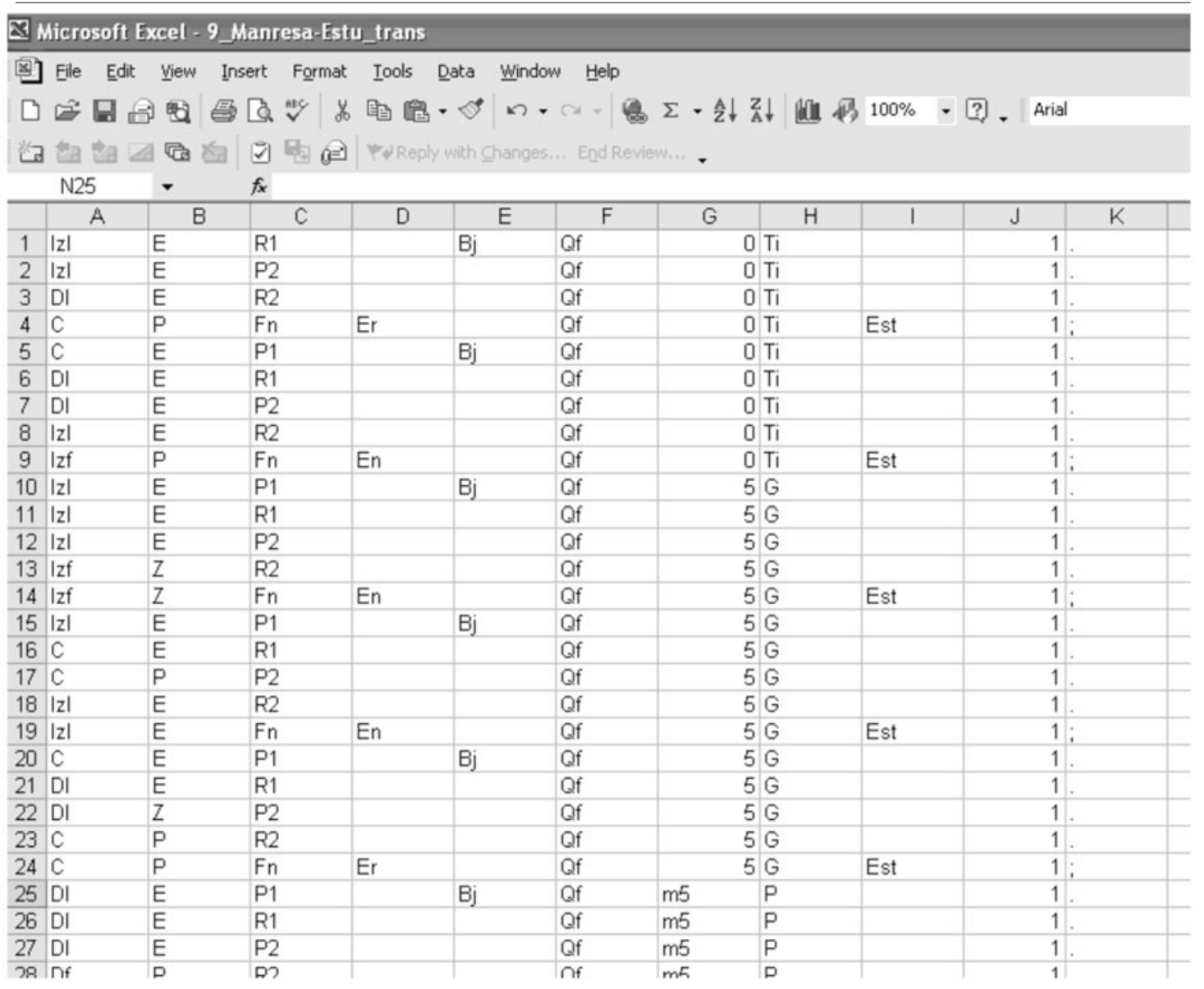

an analysis of temporal patterns (Magnusson, 1996, 2000) for our data set, using THEME software. This enabled us to obtain and represent the dendograms corresponding to actions composed of concurrent codes, with the temporal distance between their occurrence (Anguera, 2005) remaining relatively invariant within the previously fixed critical interval.

A total of 3,478 T-patterns were detected in the subset of games won. From all the criteria available in our observational device, we selected some of them to mix for the analysis of temporal patterns. In the first analysis, we selected four criteria: zone, laterality, action, and ending. For the second analysis, we selected seven criteria: zone, laterality, action, court, ending, game, and beginning of the play. After obtaining all the T-patterns, we chose the most significant ones in order to explain the dynamics of the observed team during each of these five won games (see Figure 5).

All the shots taken from the center paint spot are noteworthy. On the one hand, the missed ones come from an R2 on the DfP (right baseline paint spot), which means a oneon-one on the low post, mostly by the power forwards and centers. On the other hand, the makes come from an R2 on the same spot, CP, which we interpret as a one-on-one and an extra pass when the assists close up. One-on-ones are executed by inside players (power forwards and centers) or outside players (guards and forwards) (see Figure 6).

This temporal pattern helps to understand the mistakes made by the observed team as regards the outside shooting percentages. They kept passing the ball in the same spots, by using hand-offs or regular passes, but without swinging the ball completely (just from the center spot to the left or vice versa), and the defense was always well situated; they therefore missed every time (see Figure 7).

These patterns show the new mixed criteria and reveal fast breaks executed down the central lane of the court. Actions 14, 15, and 16 show a proper swing of the ball and one-on-ones played from the DLE (right-side outside) and finished successfully on the center paint spot. Actions 1 and 2 describe a short pass or a hand-off on the right-side outside. Actions 3, 4, 5, and 6 produced an important pattern that was repeated many times during the five games, and which shows a mistake made after the observed team swings the ball. 
Table 5

Example of a Transformed Recording That Has Been Exported to the THEME Software (Game Between Manresa and Unicaja), With Data in .CSV Format

\begin{tabular}{|c|c|c|c|c|c|}
\hline & A & B & C & D & $E$ \\
\hline 1 & DATANAME & $\mathrm{T}$ & event & & \\
\hline 2 & primer & 10946 & & & \\
\hline 3 & primer & 10955 & $\mathrm{DI}, \mathrm{E}, \mathrm{P} 1, \mathrm{Bj}, \mathrm{Qf}$ & & \\
\hline 4 & primer & 10956 & DI,E,R1,Qf & & \\
\hline 5 & primer & 11046 & $C, E, P 2, Q f$ & & \\
\hline 6 & primer & 11069 & $\mathrm{Df}, \mathrm{P}, \mathrm{R} 2, \mathrm{Qf}$ & & \\
\hline 7 & primer & 11080 & $\mathrm{Df}, \mathrm{P}, \mathrm{Fn}, \mathrm{En}, \mathrm{Q}$ & $f, 2 h, E s t$ & \\
\hline 8 & primer & 11186 & $Z L, Z Z, Z A C, Z F$ & $Z, Z J_{1} Z C_{1}$ & \\
\hline 9 & primer & 11745 & $2 v$ & & \\
\hline 10 & primer & 11802 & ZL,ZZ,ZAC, ZF & $Z \mathrm{ZJ}, \mathrm{ZC}, 2$ & \\
\hline 11 & primer & 11785 & $C, F u, P 2, S f, D$ & & \\
\hline 12 & primer & 11793 & Izf,P,R2,Def & & \\
\hline 13 & primer & 11957 & $C, P, F n, E r, Q f$ & Est & \\
\hline 14 & primer & 11986 & ZL,ZZ,ZAC,ZF & $Z J, Z C, Z$ & \\
\hline 15 & primer & 12202 & $2 v$ & & \\
\hline 16 & primer & 12227 & ZL,ZZ,ZAC,ZF & $Z \mathrm{ZJ}, \mathrm{ZC}, 2$ & \\
\hline 17 & primer & 12510 & $\mathrm{DI}, \mathrm{E}, \mathrm{P} 1, \mathrm{Bj}, \mathrm{Qf}$ & & \\
\hline 18 & primer & 12519 & $C, E, R 1, Q f$ & & \\
\hline 19 & primer & 12599 & $|z|, E, P 2, Q f$ & & \\
\hline 20 & primer & 12620 & $C, E, R 2, Q f$ & & \\
\hline 21 & primer & 12666 & $C, P, F n, E r, Q f$ & Est & \\
\hline 22 & primer & 12712 & $Z L, Z Z, Z A C, Z F$ & $Z J, Z C, Z$ & \\
\hline 23 & primer & 14276 & $\mathrm{Df}, \mathrm{P}, \mathrm{Rd}, \mathrm{Bj}, \mathrm{De}$ & & \\
\hline 24 & primer & 14285 & Df,P,P1,Def & & \\
\hline 25 & primer & 14294 & C,P,R1,Def & & \\
\hline 26 & primer & 14471 & Izf,P,P2,Qf & & \\
\hline 27 & primer & 14497 & $C, Z, R 2, Q f$ & & \\
\hline 28 & primer & 14506 & $\mathrm{C}, \mathrm{Z}, \mathrm{Fn}, \mathrm{Er}, \mathrm{Qf}$ & Est & \\
\hline
\end{tabular}

\section{Detecting Patterns of Behavior}

In order to carry out a robust data analysis, we conducted a retrospective lag sequential analysis using the SDIS-GSEQ software, Version 4.1.5 (Bakeman \& Quera, $1992,2001)$, the main goal being to detect any regularities between all the occurred behaviors (Qf, P2, and Df) and the conditioned behaviors that appear during the retrospective sequential patterns (see Table 6).

We considered the occurrences that happened in both directions, indicated in the figures (right side) through double arrows, and also calculated the adjusted residuals in relation to the negative lags (from R-1 to R-5), which show the actions that occur consistently before each behavior (see Figure 8).

The given behavior in this sequence is Qf (offensive court), and it has a strong occurrence during the 0 lag with D1, P2, Z, R2, Df, and P. This series shows that many plays are registered on the offensive court, meaning that the observed team constructs its plays during a positional five-on-five (see Figure 9).

P2 shows a strong relationship, at the same time (lag 0), with Df, P, C, and Z. Thus, P2 occurs at the same time on the paint, central spot, and right baseline. With lag 1 there is a significant relationship with Z, Df, and P. All the actions occurring previously take place on the same spot or on the spots nearby (see Figure 10).

In this relationship between these categories, the starting point is the Df category (right baseline). This is the one that occurs most often with $\mathrm{P}(\operatorname{lag} 0)$, inside the paint. The pass comes from the outside spots or zone spots (right side), as can be seen in lag 5. There is also a strong connection between the inside spots Df and $\mathrm{P}$, for lags 0 and 1. All these actions close to the rim succeed each other, thus providing privileged zones from which to try to score.

\section{DISCUSSION}

It is particularly interesting to analyze the endings of basketball plays through the complementary use of three procedures: descriptive analysis of actions, detection of T-patterns, and analysis of behavioral patterns. This methodology has enabled us to evaluate the play of the observed team according to the zones in which actions take place, 


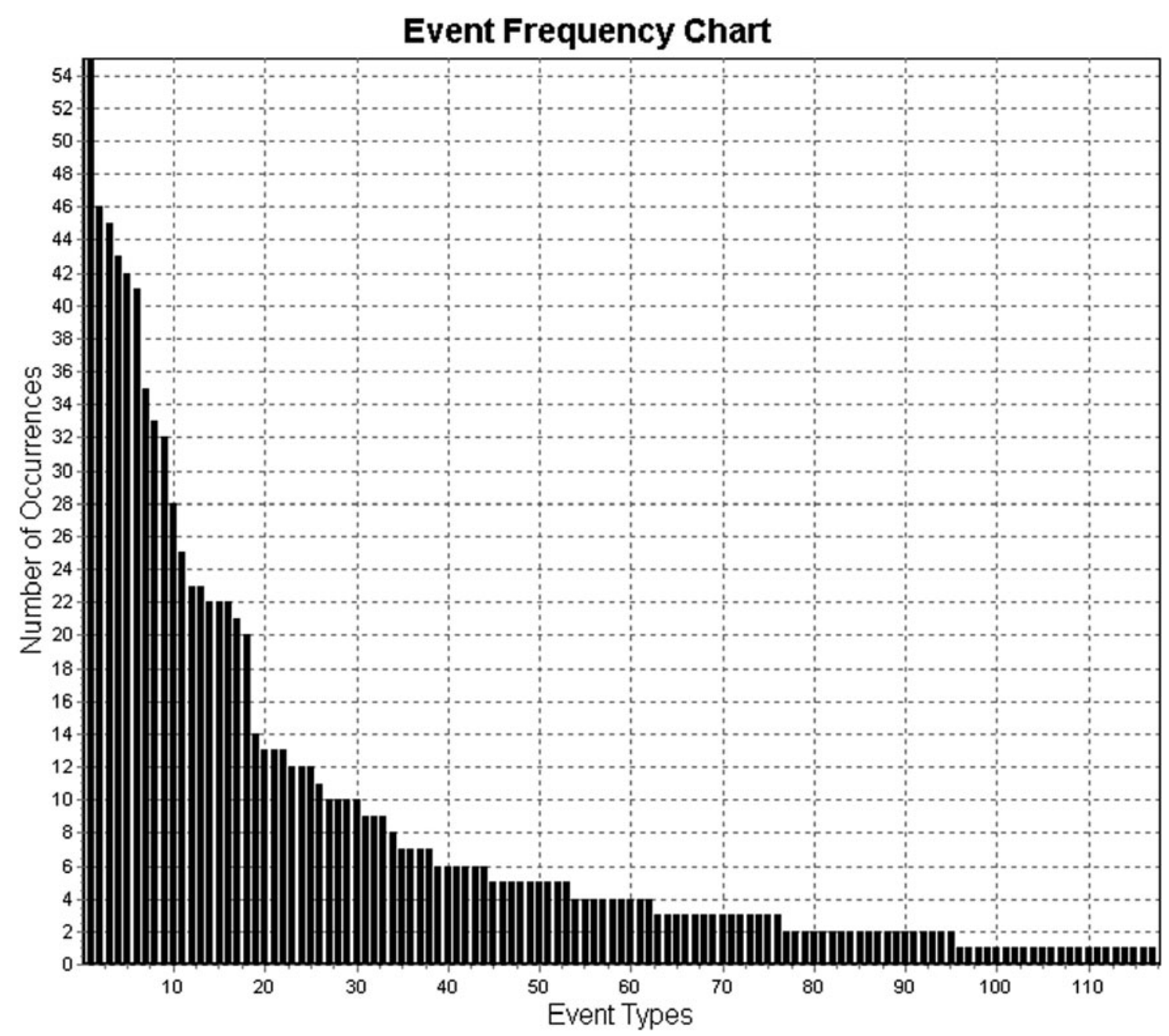

Figure 4. Plot of the first T-pattern of the 677 corresponding to games lost.

the temporal relationships between them, and the dependency relationships between the established criteria.

Thus, we have been able to assess two aspects: the effectiveness of offensive plays, which reflects factors that are important for the team's success and occurred in all five games won; and erroneous collective actions, or team mistakes, that can be improved upon and perfected.

It can be stated that in the five games won, (1) the observed team establishes a scoring equilibrium between outside and inside zones. In this case, 72 points were scored from beyond the three-point line and 110 from the paint. (2) For all the inside balls that ended in a basket, the observed team receives the last pass from Df, leading to a one-and-one and finishing in the center of the paint. Furthermore, the balls ending in CP come from assists with R2 in the same zone. (3) Outside movement of the ball is often not good as the observed team positions itself in outside zones (E) that are very close to one another, or even in the same zone (hand to hand), and many shots fail due to poor movement-in other words, a shot when the defense is well positioned. (4) Successful counterattacks take place after a player has received the last pass in CP, finishing in the same zone.

\section{CONCLUSIONS}

This study has considered scoring percentages, successful zones, significant ball movements that lead to a basket, significant ball movements that end in a mistake, and counterattacks. The relative identification of these offensive plays in basketball has been possible through the use of systematic observation.

The data analysis entailed the combined use of an initial descriptive analysis and an analysis of behavior patterns (T-patterns) using the THEME software and a lag sequential analysis using the SDIS-GSEQ software. The detection of behavior patterns in offensive plays (T-patterns) proves to be particularly interesting and revealing, it being consistent with the retrospective lag analysis of these plays. We have found that this procedure of comparative and su- 

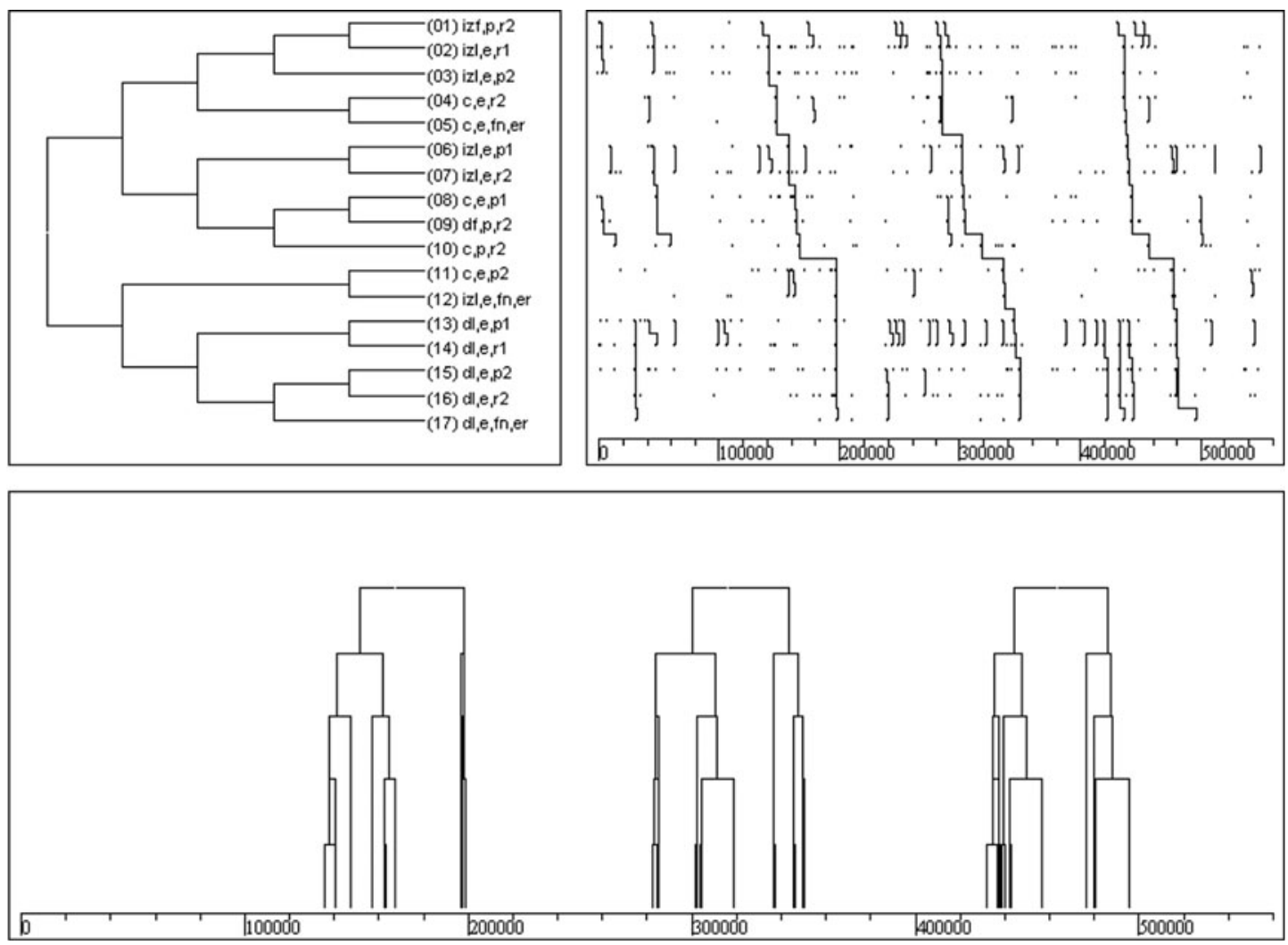

Figure 5. Plot of the first T-pattern of the $\mathbf{7 7 3}$ corresponding to games won.
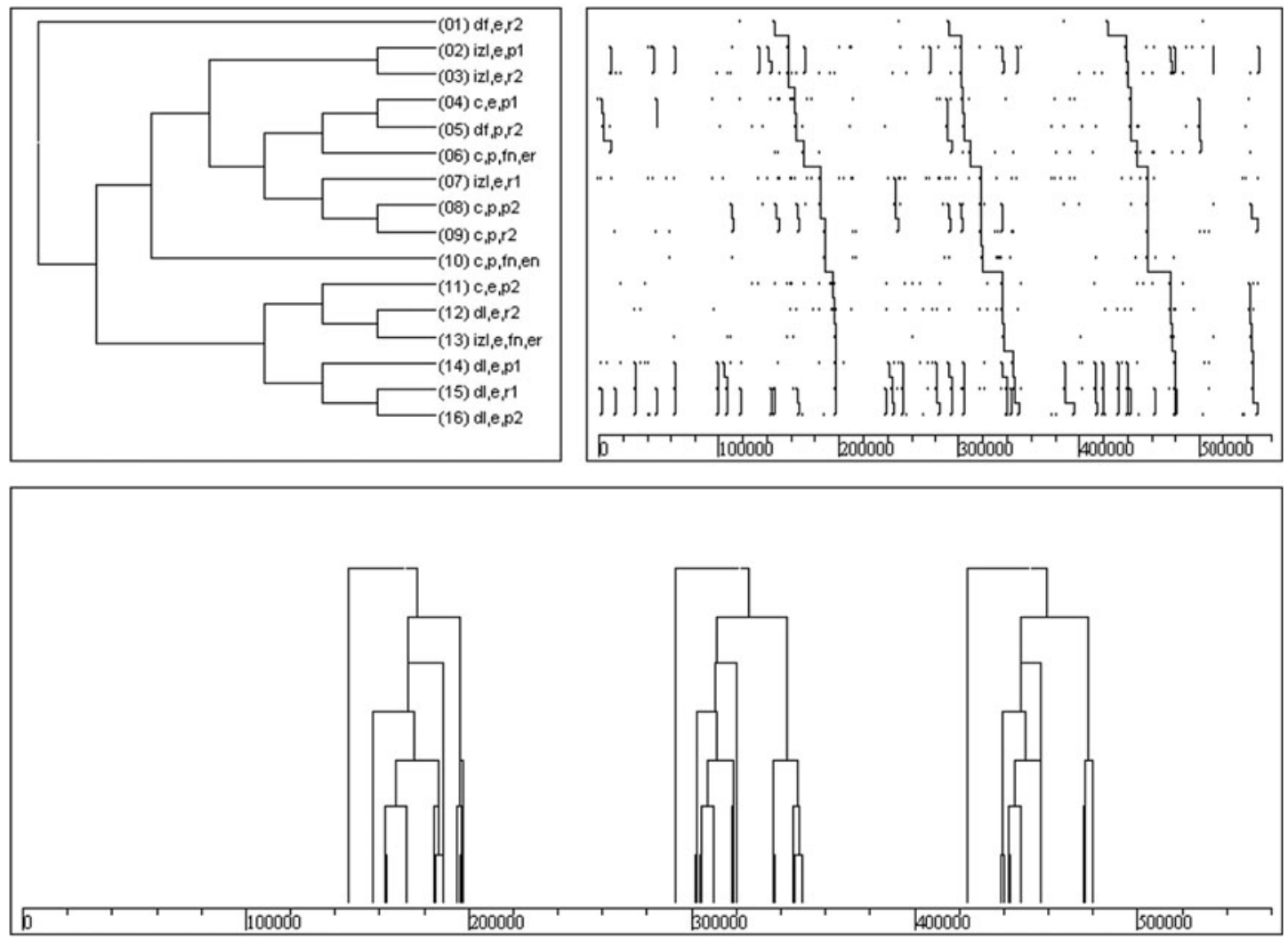

Figure 6. Plot of the first T-pattern of the 750 corresponding to games won. 

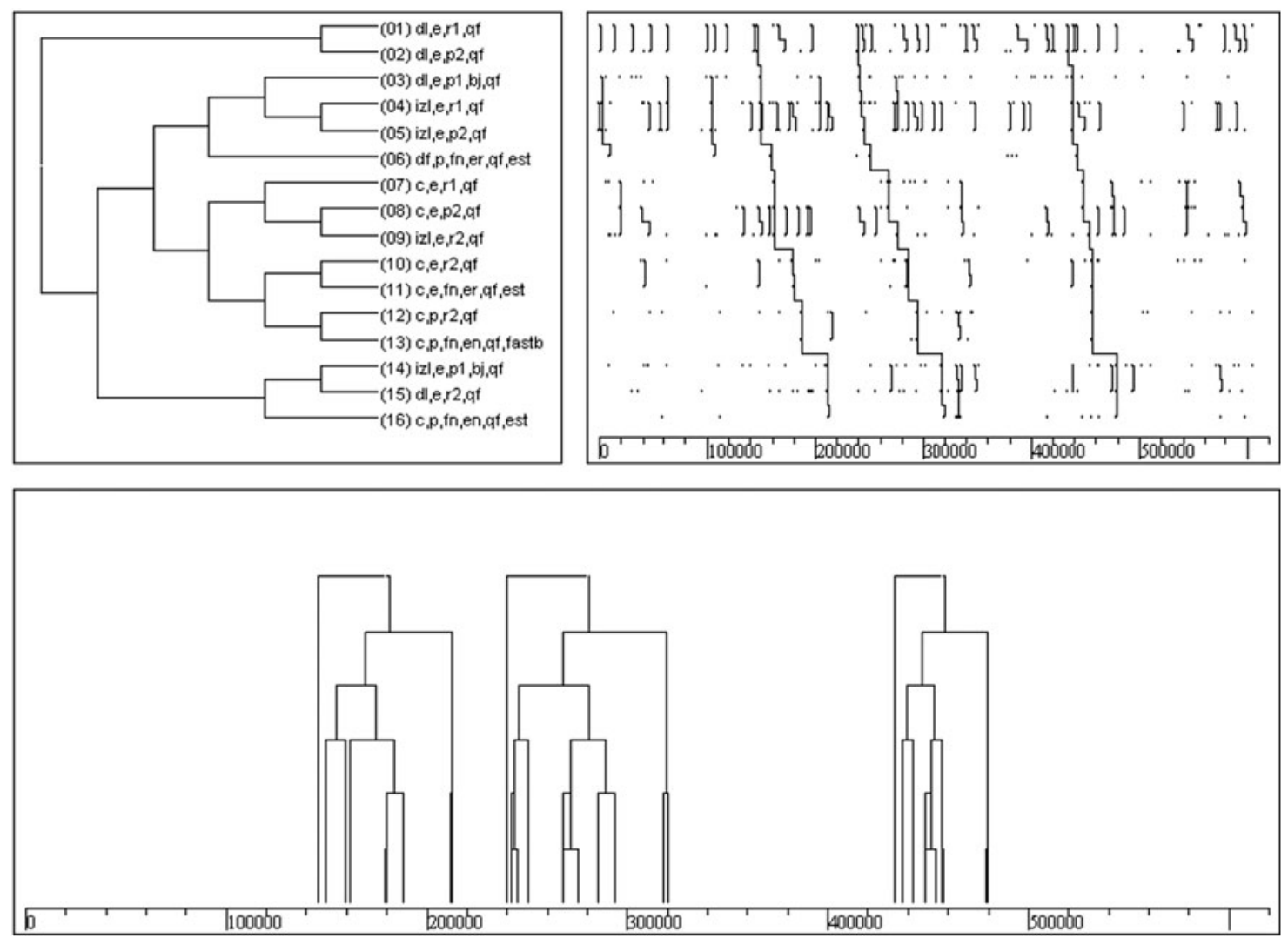

Figure 7. Plot of the first T-pattern of the 677 corresponding to games lost.

perimposed analysis is useful for evaluating the offensive play of the observed team, and this therefore underlines the suitability of the analyses carried out.

As regards future studies, the present design serves as a methodological example, since the criteria can be estab- lished and mixed according to the needs of each researcher. In subsequent research, we aim to use the observed team's own plays as the criterion to be broken down further, thereby giving play more meaning, and thus analyze and evaluate in greater depth the offensive play of this team.

Table 6

Adjusted Residuals Calculated by Means of Lag Sequential Analysis (R-5 to R0) Using the Given Behaviors Qf, P2, and Df

\begin{tabular}{rrrrrrr}
\hline & R-5 & R-4 & R-3 & R-2 & \multicolumn{1}{c}{ R-1 } & \multicolumn{1}{c}{ R0 } \\
\hline Qf & Qf 5.266 & Df 2.911 & E 2.540 & & R1 2.637 & P2 13.172 \\
& & C 3.037 & R1 3.856 & & Qf 8.072 & D1 5.653 \\
& & & & & & Z 6.238 \\
& & & & & & R2 13.324 \\
& & & & & & Df 8.146 \\
& & & & & Z 3.201 & Z 2.385 \\
P2 & R1 2.946 & E 2.622 & & & Df 5.006 & Df 4.238 \\
& & & & & P 7.515 & P 0.864 \\
& & & & Qf 1.976 & Qf 1.978 & P 11.766 \\
& & & & & Df 8.055 & \\
\hline
\end{tabular}




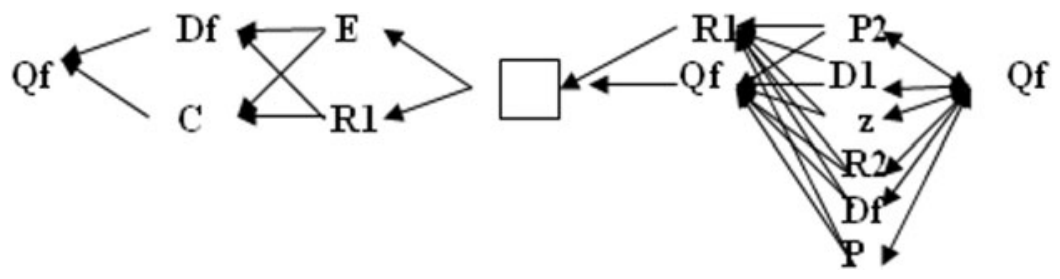

Figure 8. Retrospective lag sequential pattern corresponding to the given behavior Qf.

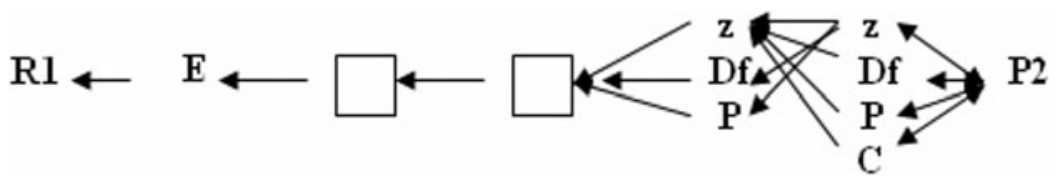

Figure 9. Retrospective lag sequential pattern corresponding to the given behavior $\mathbf{P 2}$.

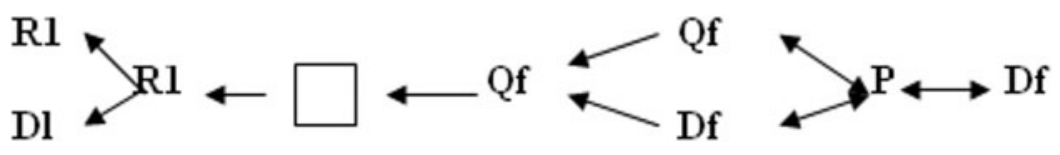

Figure 10. Retrospective lag sequential pattern corresponding to the given behavior Df.

\section{AUTHOR NOTE}

We gratefully acknowledge the support of the Spanish government project Avances tecnológicos y metodológicos en la automatización de estudios observacionales en deporte (Dirección General de Investigación, Ministerio de Ciencia e Innovación), Grant PSI2008-01179. Correspondence concerning this article should be addressed to O. Camerino, INEFC Lleida, University of Lleida, Lleida, Spain (e-mail: ocamerino@, inefc.udl.cat).

\section{REFERENCES}

Anguera, M. T. (2005). Microanalysis of T-patterns: Analysis of symmetry/asymmetry in social interaction. In L. Anolli, S. Duncan, M. Magnusson, \& G. Riva (Eds.), The hidden structure of social interaction: From genomics to culture patterns (pp. 51-70). Amsterdam: IOS Press.

Anguera, M. T., Blanco-Villaseñor, A., \& Losada, J. L. (2001). Diseños observacionales, cuestión clave en el proceso de la metodología observacional. Metodología de las Ciencias del Comportamiento, $\mathbf{3}$, 135-161.

Anguera, M. T., \& Jonsson, G. K. (2003). Detection of real-time patterns in sport: Interactions in football. International Journal of Computer Science in Sport [Online journal], 2, 118-121.

BAKEMAN, R., \& QuERA, V. (1992). SDIS: A sequential data interchange standard. Behavior Research Methods, Instruments, \& Computers, 24, 554-559.

BaKeman, R., \& Quera, V. (2001). Using GSEQ with SPSS. Metodología de las Ciencias del Comportamiento, 3, 195-214.

BANDURA, A. (1977). Self-efficacy: Toward a unifying theory of behavioural change. Psychological Review, 84, 191-215.

Bandura, A. (1982). Self-efficacy mechanism in human agency. American Psychologist, 37, 122-147.

BANDURA, A. (1986). Social foundations of thought and action: A social cognitive theory. Englewood Cliffs, NJ: Prentice Hall.

Bandura, A. (1997). Self-efficacy: The exercise of control. New York: Freeman.
Blanco-Villaseñor, A., \& Anguera, M. T. (2000). Evaluación de la calidad en el registro del comportamiento: Aplicación a deportes de equipo. In E. Oñate, F. García-Sicilia, \& L. Ramallo (Eds.), Métodos numéricos en ciencias sociales (pp. 30-48). Barcelona: Centro Internacional de Métodos Numéricos en Ingeniería.

Bloomfield, J., Jonsson, G. K., Polman, R., Houlahan, K., \& O'Donoghue, P. (2005). Temporal patterns analysis and its applicability in soccer. In L. Anolli, S. Duncan, M. Magnusson, \& G. Riva (Eds.), The hidden structure of social interaction: From genomics to culture patterns (pp. 237-251). Amsterdam: IOS Press.

Borrie, A., Jonsson, G. K., \& Magnusson, M. S. (2001). Application of T-pattern detection and analysis in sports research. Metodología de las Ciencias del Comportamiento, 3, 215-226.

Borrie, A., Jonsson, G. K., \& Magnusson, M. S. (2002). Temporal pattern analysis and its applicability in sport: An explanation and exemplar data. Journal of Sports Sciences, 20, 845-852.

Castellano, J., Perea, A., Alday, L., \& Hernández Mendo, A. (2008). The measuring and observation tool in sports. Behavior Research Methods, 40, 898-905.

FeLtz, D. L. (1992). Understanding motivation in sport: A self-efficacy perspective. In G. C. Roberts (Ed.), Motivation in sport and exercise (pp. 93-106). Champaign, IL: Human Kinetics.

Garganta, J. (2001). Análisis del juego en el fútbol: Recorrido evolutivo de las concepciones, métodos e instrumentos. Revista de Entrenamiento Deportivo, XIV, 5-14.

George, T. R., \& Feltz, D. L. (1995). Motivation in sport from a collective efficacy perspective. International Journal of Sport Psychology, 26, 98-116.

Jonsson, G. K., Anguera, M. T., Blanco-Villaseñor, A., Losada, J. L., Hernández-Mendo, A., ARdÁ, T., ET AL. (2006). Hidden patterns of play interaction in soccer using SOF-CODER. Behavior Research Methods, 38, 372-381.

Jonsson, G. K., BJarkadottir, S. H., Gislason, B., Borrie, A., \& MaGnusson, M. S. (2003). Detection of real-time patterns in sports: Interactions in football. In C. Baudoin (Ed.), L'éthologie appliquée aujourd'hui [Applied ethology today]: Vol. 3. Ethologie humaine (pp. 35-75). Levallois-Perret, France: Editions ED. 
Magnusson, M. S. (1996). Hidden real-time patterns in intra- and interindividual behavior. European Journal of Psychological Assessment, 12, 112-123.

Magnusson, M. S. (2000). Discovering hidden time patterns in behavior: T-patterns and their detection. Behavior Research Methods, Instruments, \& Computers, 32, 93-110.

Prapavessis, H., \& Carron, A. V. (1996). The effect of group cohesion on competitive state anxiety. Journal of Sport \& Exercise Psychology, 18, 64-74.
Riedl, R., Brandstätter, E., \& Roithmayr, F. (2008). Identifying decision strategies: A process- and outcome-based classification method. Behavior Research Methods, 40, 795-807.

Widmeyer, W. N., Brawley, L. R., \& Carron, A. V. (1992). Group dynamics in sport. In T. S. Horn (Ed.), Advances in sport psychology (pp. 163-180). Champaign, IL: Human Kinetics.

(Manuscript received October 30, 2008; accepted for publication November 18, 2008.) 